\title{
Confirmatory Factor Analysis of the Stagnation Scale-A Traditional Chinese Medicine Construct Operationalized for Mental Health Practice
}

\author{
Siu-man Ng • Ted Chun Tat Fong • Xiao-lu Wang • \\ Yi-jie Wang
}

Published online: 31 January 2011

(C) The Author(s) 2011. This article is published with open access at Springerlink.com

\begin{abstract}
Background Traditional Chinese medicine stagnation ("yu") syndrome is characterized by a cluster of mind/body obstruction-like symptoms. Previous studies have operationalized the concept as a psychological construct through scale development, producing a three-factor 16-item inventory with good psychometric properties.

Purpose The study aimed to further validate the Stagnation Scale by confirmatory factor analysis (CFA) and examine self-appraisal of stagnation as an illness.

Method A cross-sectional questionnaire survey was conducted on a random community sample of 755 adults recruited by cluster sampling in Hong Kong.

Results CFA revealed a good fit of the three-factor model $(\mathrm{CFI}=.95 ; \mathrm{RMSEA}=.077$; SRMR $=.043)$. ROC analysis suggested a cutoff score at 50 on stagnation total score for predicting self-appraisal of an illness condition, with false positive and negative rates at $25.8 \%$ and $23.3 \%$, respectively. Overall, $6.2 \%$ participants self-appraised to suffer stagnation symptoms to a degree of an illness, and for it, $1.9 \%$ participants intended to seek treatment. Stagnation showed positive correlations with physical distress, depression, and anxiety $(r=.59-.76, p<.01)$ and negative correlation with age $(r=-.22, p<.01)$.

Conclusion The Stagnation Scale appeared to be robust in factorial and construct validity. With prevalence of illness by self-appraisal at $6.2 \%$ and intention for treatment at $1.9 \%$, stagnation is a fairly common condition associated with treatment-seeking behaviors.
\end{abstract}

S.-m. Ng • T. C. T. Fong $(\bowtie) \cdot$ X.-1. Wang $\cdot$ Y.-j. Wang Department of Social Work \& Social Administration and Centre on Behavioral Health, The University of Hong Kong,

Pokfulam,

Hong Kong SAR, People's Republic of China

e-mail: ttaatt@hku.hk
Keywords Stagnation - Scale validation .

Confirmatory factor analysis · Cutoff

\section{Introduction}

The English translation of the "yu" concept of traditional Chinese medicine (TCM) is "stagnation" [1]. Literally, "yu" in Chinese means entangled, not flowing freely, and restrained stretching. Stagnation syndrome is an old diagnostic entity of TCM which was first depicted in medical classics written some 2,000 years ago. The syndrome is characterized by a cluster of mind/body obstruction-like symptoms such as pent-up emotions, feeling something stuck inside the head, throat, and chest, indigestion, bowel dysfunctions, and abdominal distension [2]. Having a prominent somatic component, stagnation syndrome carries little mental disorder notion. Because of its long history and high social acceptability, stagnation syndrome is deeply embedded into Chinese culture, being a health condition which is often openly discussed, as well as a common reason for seeking attention and help.

With a strong systemic orientation, TCM emphasizes mind/body connection and considers the interactions between repressed emotions and dysfunction of the liver meridian the central mechanism for the onset and maintenance of the stagnation syndrome. It often begins with repression of emotions, and in particular, repression of anger is the most common. While the precipitating events trigger the onset, psycho-spiritual predisposing factors play an instrumental role in shaping the behavioral reactions. In this context, overattachment is a notable notion in the psycho-spiritual domain. While it overlaps with the notion of dysfunctional thoughts in the cognitive domain, overattachment expands to the spiritual domain and is 
related to existential issues. It is not only about judgments of good and bad, but is also about fear of loss, non-acceptance of impermanence, and struggles between the personal and transpersonal self. Unable to transcend and let go of, overattachment leads to entangled, clogged emotions and restrictive behavioral expressions. From systemic perspective, causes and results can hardly be differentiated from each other. Psycho-spiritual and bio-behavioral actions mutually reinforce each other, contributing to a cluster of symptoms characterizing the stagnation syndrome.

In view of the potential value of the TCM stagnation concept in modern mental health practice, in particular, in the context of Chinese culture, we have attempted to operationalize the construct through scale development [3, 4]. A multidisciplinary team of psychiatrist, psychiatric social worker, clinical psychologist, and TCM practitioner was formed and adopted a concept-driven approach to identify the essential domains of stagnation and generate the item pool. After repeated deliberations, consultations, and pilots, a pool of 63 candidate items was constructed. Subsequent exploratory factor analysis on a sample of 603 adult Chinese revealed a three-factor 16-item solution which demonstrated robust factor structure, good criterion-related and construct validity, and high internal consistency. Cronbach's alpha of the entire scale was 0.91 . The three subscales had Cronbach's alpha ranged between 0.82 and 0.88 . Meaningful loadings of the 16 items on their respective factor ranged between 0.61 and 0.79 , and there were no double loadings. Correlation with the criterion validity checking item was 0.71 .

The three factors were named as Overattachment, BodyMind Obstruction, and Affect-Posture Inhibition. In Overattachment, the themes are preoccupation of fear of losing xwhat one possesses, being not as accomplished as others, and being unable to let go of some matters. In Body-mind Obstruction, the theme is somatic obstruction-like symptoms such as a feeling that the stomach is clogged and something is obstructing the throat. In Affect-Posture inhibition, the themes are being overly self-conscious, heightened awareness and uneasiness, resulting in inhibited facial expression of affect and postural movement.

A ten-point anchored numeric scale was adopted as the response format to the 16 items, where " 1 " indicated "has not occurred at all" and "10" indicated "occurring every single moment". The composite stagnation score is the sum of all 16 individual item scores, yielding a theoretical range of 16-160. The score showed significant positive correlations with Beck Depression Inventory [5] and the 12-item General Health Questionnaire [6] at moderate magnitude. Stagnation and depression had different correlation patterns with demographic data. In contrary to depression, stagnation showed no significant difference between genders and appeared to be significantly more prevalent among adults who are younger, better educated, and occupying professional or managerial positions. These findings suggest that stagnation is a distinct syndrome distinguishable from depression in conceptualizations, clinical presentations, and demographic pattern.

In view of the satisfactory psychometric properties revealed and the potential clinical applications of the construct, the current study aimed to further validate the Stagnation Scale by confirmatory factor analysis. By recruiting a random community sample, another key objective was to evaluate self-appraisal of stagnation as an illness condition and the associated consultation behaviors.

\section{Methods}

\section{Subjects}

A cross-sectional questionnaire survey on a random community sample was conducted. Cluster sampling method was adopted for tighter quality control and ease of pursuing follow-up studies in the future. Totally, 13 residential blocks of public and private housing estate in the Aberdeen District of Hong Kong were selected for the study. The Aberdeen District was comparable with the whole population of Hong Kong in terms of household size (median at 3.0) and income (median at HK\$20,500 per month; 1 US\$=7.8 HK\$) according to the 2006 census data [7]. In each selected cluster, that was a residential block, $100 \%$ sampling was performed. All households were invited to participate in the study by means of a letter followed by a home visit. Target participants were adults residing in the household and aged between 18 and 65. Inclusion criteria were: (1) able to communicate in Chinese (Cantonese or Putonghua) and (2) able to understand and respond to the questionnaire, which is in Chinese, in either self-report or interviewer-administered manner. The study was approved by the Ethical Committee of the Faculty of Social Sciences, The University of Hong Kong.

\section{Measures}

The questionnaire included basic demographic data, the Stagnation Scale, and a battery of validation scales, namely, the 12-item General Health Questionnaire (GHQ-12) [6, 8], Hospital Anxiety and Depression Scale (HADS) [9-11], and the Physical Distress subscale of the Body-Mind-Spirit Well-Being Inventory (BMSWBI) [12].

GHQ-12 [8] is a self-administered screening instrument for common mental disorders in community settings, especially depression and anxiety disorders. It has been widely used in community and primary care settings, and its measurement properties have been studied in diverse cultural environments [13]. The Chinese version has been validated and found to have satisfactory psychometric 
properties, with sensitivity and specificity of $86 \%$ and $80 \%$, respectively $[6,14]$. Using the $0-1-2-3$ coding, possible scores ranged between 0 and 36 . The higher the score, the more likely a case of mental health problem is.

HADS measures anxiety and depression symptoms in both clinical and community settings [9]. This is a 14-item self-report questionnaire, with seven items for anxiety and seven items for depression. A Chinese version has been developed and validated in Hong Kong [10, 11]. It has good psychometric properties and has been used in many local mental health-related studies. The scale produces two scores, namely, depression and anxiety scores. Each score has a theoretical range of $0-21$, and the higher the score, the worse the mental state is. Suggested reference cutoff points are: $0-7$ as "normal", $8-10$ as "mild", $11-14$ as "moderate", and 15-21 as "severe" [9].

The Physical Distress subscale of BMSWBI measures subject distress of multiple somatic symptoms [12]. The 14-item self-report questionnaire has been developed and validated with Chinese adults in Hong Kong. It has high internal consistency, with Cronbach's alpha at 0.87 . The scale produces a single score which has a theoretical range of $0-140$. The higher the score, the more distressful the physical discomforts are.

Participants' subjective appraisal of stagnation symptoms as an illness condition was inquired by self-constructed items. Firstly, the participant was asked if he/she considered the stagnation symptoms amounted to a degree of an illness condition ("yes" or "no"). If "yes", the participant was asked whether and when he/she wanted to seek treatment ("yes and within a week", "yes and a week to a month", "yes and after a month", or "no").

\section{Statistical Analysis}

Descriptive, correlation, and regression analyses were conducted on SPSS 18.0. Confirmatory factor analysis was conducted on Mplus 5.2. Participants' subjective appraisal of illness condition was used as the criterion for exploring cutoff points on the Stagnation Scale by balancing false-positive and false-negative rates. Participants self-appraised as ill and not-ill were compared by $t$ test on continuous variable measures and chi-square test on nominal variable measures.
Table 1 Comparisons of profiles of participants self-appraised as suffering from stagnation symptoms at an illness condition versus participants self-appraised not at an illness condition $t t$ test value, $G H Q$ General

Health Questionnaire, BMSWBI

Body-Mind-Spirit Well-Being Inventory, HADS Hospital Anxiety and Depression Scale ${ }^{*} p=0.05 ; * * p=0.01$

\begin{tabular}{|c|c|c|c|c|c|c|}
\hline & \multicolumn{2}{|c|}{ Self-appraised not ill $(N=699)$} & \multicolumn{2}{|c|}{ Self-appraised ill $(N=46)$} & \multirow[b]{2}{*}{$\chi^{2}$} & \multirow[b]{2}{*}{$p$} \\
\hline & $N$ & $\%$ & $N$ & $\%$ & & \\
\hline \multicolumn{7}{|l|}{ Gender } \\
\hline Male & 310 & 44.4 & 12 & 26.1 & \multirow[t]{2}{*}{5.90} & \multirow[t]{2}{*}{$.02 *$} \\
\hline Female & 388 & 55.6 & 34 & 73.9 & & \\
\hline \multicolumn{7}{|l|}{ Marital status } \\
\hline Single & 222 & 31.9 & 12 & 26.1 & \multirow[t]{3}{*}{5.02} & \multirow[t]{3}{*}{.08} \\
\hline Married & 446 & 64.1 & 29 & 63.0 & & \\
\hline Divorced & 28 & 4.0 & 5 & 10.9 & & \\
\hline \multicolumn{7}{|l|}{ Educational level } \\
\hline Primary or below & 115 & 16.9 & 7 & 15.2 & \multirow[t]{3}{*}{1.64} & \multirow[t]{3}{*}{.44} \\
\hline Secondary & 335 & 49.2 & 27 & 58.7 & & \\
\hline Tertiary or above & 231 & 33.9 & 12 & 26.1 & & \\
\hline \multicolumn{7}{|c|}{ Personal monthly income (HK\$) } \\
\hline Below 5,000 & 44 & 6.5 & 4 & 8.9 & \multirow[t]{5}{*}{2.16} & \multirow[t]{5}{*}{.71} \\
\hline $5,000-9,999$ & 143 & 21.0 & 12 & 26.7 & & \\
\hline $10,000-19,999$ & 203 & 29.8 & 11 & 24.4 & & \\
\hline Over 20,000 & 143 & 21.0 & 7 & 15.6 & & \\
\hline \multirow[t]{2}{*}{ Not applicable } & 149 & 21.8 & 11 & 24.4 & & \\
\hline & M & SD & $\mathrm{M}$ & $\mathrm{SD}$ & $t$ & $p$ \\
\hline Age $(N=738)$ & 41.64 & 11.47 & 41.58 & 11.15 & 0.03 & .97 \\
\hline Stagnation $(N=717)$ & 39.10 & 22.40 & 80.93 & 32.42 & 8.33 & $<.01 * *$ \\
\hline GHQ-12 $(N=737)$ & 1.75 & 2.39 & 6.29 & 3.61 & 8.31 & $<.01 * *$ \\
\hline \multicolumn{7}{|l|}{ BMSWBI $(N=717)$} \\
\hline Physical distress & 22.67 & 19.39 & 43.51 & 25.31 & 5.30 & $<.01 * *$ \\
\hline \multicolumn{7}{|l|}{ HADS $(N=740)$} \\
\hline Anxiety & 5.20 & 3.53 & 10.60 & 3.10 & 9.89 & $<.01 * *$ \\
\hline Depression & 4.27 & 3.10 & 8.07 & 3.14 & 8.03 & $<.01 * *$ \\
\hline
\end{tabular}




\section{Results}

Totally, 755 completed questionnaires were collected, with $43.3 \%$ men and $56.7 \%$ women. Mean age of the respondents was 41.7 (standard deviation (SD), 11.5). Regarding marital status, percentages being single, married, and separated were $31.1 \%, 63.8 \%$, and $4.5 \%$, respectively. Educational level was $16.4 \%$ at primary level or below, $49.0 \%$ at secondary level, and $32.2 \%$ at tertiary level.

Confirmatory factor analysis was performed on the Stagnation Scale with the dataset. For the original threefactor 16-item structure, comparative fit index (CFI), root mean square error of approximation (RMSEA), and standardized root mean square residual (SRMR) were found to be $.94, .085$ and .040 , respectively. If residual covariance between item 15 and 16, which showed high correlation, was allowed, the resultant model fit was adequate with $\mathrm{CFI}=.95, \mathrm{RMSEA}=.077,90 \% \mathrm{CI}$ of $\mathrm{RMSEA}=.071-.083, \mathrm{SRMR}=.043$, respectively.

Regarding construct validity, correlational analyses of the Stagnation Scale with validation scales were performed. The correlations of Stagnation scores with Physical Distress on
BMSWBI, GHQ-12, and the anxiety and depression scores on HADS were $.59, .69, .76$, and .60 , respectively (all $p<.01$ ).

Using participant's subjective appraisal of illness condition as the criterion, ROC analysis was carried out to evaluate the optimal cutoffs on the stagnation score with an aim to balance false-positive and false-negative rates in predicting illness condition by self-appraisal. The optimally balanced cutoff points on the Stagnation Scale were revealed to be 50 for total score (false-positive and -negative rates, .258 and .233, respectively), 27 on Overattachment subscale (false-positive and -negative rates, .217 and .233), 14 on Body-Mind Obstruction subscale (false-positive and -negative rates, .212 and .233), and 10 on Affect-Posture Inhibition (false-positive and -negative rates, .242 and .256).

Regarding association with demographic data, stagnation showed a significant negative correlation with age $(r=-.22, p<.01)$. Stagnation score showed no significant statistical difference between genders or among different groups of marital status, education level, and income. Overall, $6.2 \%$ of the participants (46 out of 745) considered themselves suffering from stagnation symptoms of a degree at an illness condition. Table 1 compares the
Table 2 Comparisons of profiles of participants self-appraised as suffering from stagnation symptoms at an illness condition and intended to seek treatment versus participants self-appraised as ill but have no intention to seek treatment
$U$ Mann-Whitney $U$ test value, GHQ General Health Questionnaire, BMSWBI BodyMind-Spirit Well-Being Inventory, HADS Hospital Anxiety and Depression Scale ${ }^{*} p=0.05$ level

\begin{tabular}{|c|c|c|c|c|c|c|}
\hline & \multicolumn{2}{|c|}{$\begin{array}{l}\text { Self-appraised ill with no intention } \\
\text { for treatment }(N=32)\end{array}$} & \multicolumn{2}{|c|}{$\begin{array}{l}\text { Self-appraised ill and intended for } \\
\text { treatment }(N=14)\end{array}$} & \multirow[b]{2}{*}{$\chi^{2}$} & \multirow[b]{2}{*}{$p$} \\
\hline & $N$ & $\%$ & $N$ & $\%$ & & \\
\hline \multicolumn{7}{|l|}{ Gender } \\
\hline Male & 9 & 28.1 & 3 & 21.4 & \multirow[t]{2}{*}{.23} & \multirow[t]{2}{*}{.63} \\
\hline Female & 23 & 71.9 & 11 & 78.6 & & \\
\hline \multicolumn{7}{|l|}{ Marital status } \\
\hline Single & 11 & 34.4 & 1 & 26.1 & \multirow[t]{3}{*}{3.75} & \multirow[t]{3}{*}{.15} \\
\hline Married & 18 & 56.3 & 11 & 63.0 & & \\
\hline Divorced & 3 & 9.4 & 2 & 10.9 & & \\
\hline \multicolumn{7}{|l|}{ Educational level } \\
\hline Primary or below & 3 & 9.4 & 4 & 28.6 & \multirow[t]{3}{*}{8.16} & \multirow[t]{3}{*}{$.02^{*}$} \\
\hline Secondary & 17 & 53.1 & 10 & 71.4 & & \\
\hline Tertiary or above & 12 & 37.5 & 0 & 0 & & \\
\hline \multicolumn{7}{|c|}{ Personal monthly income (HK\$) } \\
\hline Below 5,000 & 3 & 9.4 & 1 & 7.7 & \multirow[t]{5}{*}{2.96} & \multirow[t]{5}{*}{.57} \\
\hline $5,000-9,999$ & 8 & 25.0 & 4 & 30.8 & & \\
\hline $10,000-19,999$ & 9 & 28.1 & 2 & 15.4 & & \\
\hline Over 20,000 & 6 & 18.8 & 1 & 7.7 & & \\
\hline \multirow[t]{2}{*}{ Not applicable } & 6 & 18.8 & 5 & 38.5 & & \\
\hline & M & SD & M & SD & $U$ & $p$ \\
\hline Age $(N=45)$ & 38.97 & 11.39 & 48.00 & 7.62 & 110.5 & $.02 *$ \\
\hline Stagnation $(N=43)$ & 83.06 & 28.69 & 75.41 & 41.50 & 147.0 & .29 \\
\hline GHQ-12 $(N=45)$ & 6.61 & 3.66 & 5.57 & 3.52 & 179.0 & .35 \\
\hline \multicolumn{7}{|l|}{ BMSWBI $(N=43)$} \\
\hline Physical distress & 45.41 & 23.93 & 38.00 & 29.52 & 134.5 & .25 \\
\hline \multicolumn{7}{|l|}{ HADS $(N=45)$} \\
\hline Anxiety & 11.19 & 3.89 & 9.29 & 3.93 & 155.5 & .13 \\
\hline Depression & 8.28 & 3.29 & 7.57 & 2.79 & 204.5 & .64 \\
\hline
\end{tabular}


scores in stagnation, GHQ-12, anxiety, depression, physical distress, and demographic data between the self-appraised "ill" and "not ill" groups. The self-appraised ill participants were more likely to be female and had significantly higher scores in stagnation, GHQ-12, anxiety, depression, and physical distress.

Among the 46 participants who considered their stagnation symptoms amounted to an illness condition, 14 (i.e., $30.4 \%$ of the 46 self-appraised ill or $1.9 \%$ of all the 745 participants) intended to seek treatment whereas 32 intended not. Table 2 compares the scores in stagnation, GHQ-12, anxiety, depression, physical distress, and demographic data between the self-appraised ill with intention for treatment and the self-appraised ill without intention for treatment. The intend-to-treat group was significantly higher in age and lower in educational level.

\section{Conclusion and Discussion}

Confirmatory factor analysis showed that the 16-item three-factor structure of the Stagnation Scale fitted well to the dataset (CFI $=.95$, RMSEA $=.077,90 \%$ CI of RMSEA $=.071-.083, \mathrm{SRMR}=.043$ ). The cutoffs revealed for predicting self-appraisal of stagnation as an illness condition on the total score and the three subscores had reasonable false-positive and -negative rates (ranged between .21 and .26). The strong correlations with validation scales suggested that the Stagnation Scale showed stable construct validity. Regarding associations with demographic data, the current study concurred with the previous study that stagnation showed significant negative correlation with age $(r=-.22)$. In contrary to the previous study, stagnation did not show significant a association with marital status, educational level, or income in the current study.

Overall, $6.2 \%$ of participants self-appraised themselves as suffering from stagnation symptoms of a degree at an illness condition. These people also scored significantly higher in anxiety, depression, and physical distress. Among these people, $30.4 \%$ (or $1.9 \%$ of all participants) intended to seek treatment. Age (being older) appeared to a predictor of consultation behavior. Mean age of those having intention for treatment was 48.0 years $(\mathrm{SD}=7.6)$ versus 39.0 years $(\mathrm{SD}=11.4)$ of those who had no intention for treatment.

These results suggest that stagnation is a fairly prevalent problem among Hong Kong Chinese adults. Because of its image as largely a somatic illness and its high social acceptability, stagnation can be used as an entry point for engaging people in need to mental health help. While the bodily discomforts can be the anchor, intervention can be strategically and tactfully expanded to psychosocial domains. Since stagnation has a significant correlation with depression and anxiety at moderate magnitude, stagnation can also be an alternative way in approaching people suffering from depression and anxiety who are however resistant towards the notion of mental disorder or mental health problems.

Despite an ancient root in TCM and Chinese culture, the stagnation construct is not necessarily relevant to the Chinese people only. Clustering of mind/body obstruction-like symptoms may be valid and have clinical values in other cultures. The Stagnation Scale can be a tool for further studies of the construct in different populations and ethnic groups.

A discourse of the stagnation construct and somatoform disorders may have significant theoretical and practical values. The notion of medically unexplained physical symptoms is central to somatoform disorders $[15,16]$. Since the internal conflicts between the conscious and unconscious are considered as a key mechanism of these disorders, intervention focuses very much on the psychosocial domains. In the stagnation construct, the associated physical symptoms are considered as expected and explainable by TCM systemic theories and should be relieved by both physical and psychosocial means. While a strong $\mathrm{mind} /$ body connection is recognized, the role of the unconscious in the repression of emotions has barely been considered in TCM. The understanding of the psychological process of the stagnation construct is largely grounded on an interpersonal rather than an intrapsychic framework, which may reflect a collective orientation in the Chinese culture. Further studies for delineating stagnation and somatoform disorders may enhance the understanding of the dynamic associations between physical symptoms and psychobehavioral processes in different cultures.

Acknowledgement The study was funded by the General Research Fund (Ref: HKU749708H) of the Research Grant Council, Hong Kong SAR Government.

Open Access This article is distributed under the terms of the Creative Commons Attribution Noncommercial License which permits any noncommercial use, distribution, and reproduction in any medium, provided the original author(s) and source are credited.

\section{References}

1. Yuen YC, Ren JS, Wang L, Guo KZ. Chinese-English dictionary of traditional Chinese medicine. Beijing: People's Health Publishing; 1997.

2. Cai J. Advanced textbook on traditional Chinese medicine and pharmacology vol. 1. Beijing: New World Press; 1995.

3. Ng SM. Operationalising Chinese medicine 'stagnation' concept as a psychological construct. Hong Kong J Psychiatry. 2008;18 (4 (Suppl)):52.

4. Ng SM, Chan CLW, Ho DYF, Wong YY, Ho RTH. Stagnation as a distinct clinical syndrome: comparing "yu" (stagnation) in 
traditional Chinese medicine with depression. Br J Soc Work. 2006;36:467-84.

5. Yeung AS, Howarth S, Chan R, Sonawalla S, Nierenberg A, Fava $\mathrm{M}$. Use of the Chinese version of the Beck Depression Inventory for screening depression in primary care. J Nerv Ment Dis. 2002;190:94-9.

6. Shek DT. Reliability and factorial structure of the Chinese version of the General Health Questionnaire. J Clin Psychol. 1987;43:683-91.

7. HKSAR Census and Statistics Department. Population by-census. 2006. http://www.censtatd.gov.hk. Accessed 30 Aug 2007.

8. Goldberg D. The detection of mental illness by questionnaire. London: Oxford University Press; 1972.

9. Zigmond AS, Snaith RP. The Hospital Anxiety and Depression Scale. Acta Psychiatr Scand. 1983;67:361-70.

10. Leung CM, Ho S, Kan CS, Hung CH, Chen CN. Evaluation of the Chinese version of the Hospital Anxiety and Depression Scale: a cross-cultural perspective. Int J Psychosom. 1993;40:29-34.

11. Leung CM, Wing YK, Kwong PK, Lo A, Shum K. Validation of the Chinese-Cantonese version of the Hospital Anxiety and
Depression Scale and comparison with the Hamilton Rating Scale of Depression. Acta Psychiatr Scand. 1999;100:456-61.

12. Ng SM, Yau JKY, Chan CLW, Chan CHY, Ho DYF. The measurement of body-mind-spirit well-being: toward multidimensionality and transcultural applicability. Soc Work Health Care. 2005;41(1):33-52.

13. Ustun TB, Sartorius N. Mental illness in general health care-an international study. Chichester: Wiley; 1995.

14. Goldberg DP, Gater R, Sartorius N, Ustun TB, Piccinelli M, Gureje O, Rutter C. The validity of two versions of the GHQ in the WHO study of mental illness in general health care. Psychol Med. 1997;27:191-197.

15. American Psychiatric Association. Diagnostic and statistical manual of mental disorder DSM-IV-TR. Washington D.C.: American Psychiatric Association; 2000.

16. Li CT, Chou YH, Yang KC, Yang CH, Lee YC, Su TP. Medically unexplained symptoms and somatoform disorders: diagnostic challenges to psychiatrists. J Chin Med Assoc. 2009;72(5):251-6. 